\title{
Intravitreal ranibizumab injection combined with photodynamic therapy for polypoidal choroidal vasculopathy
}

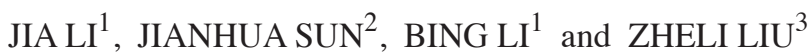 \\ ${ }^{1}$ Department of Ophthalmology, The First Affiliated Hospital of Jinzhou Medical University; ${ }^{2}$ Department of \\ Otorhinolaryngology, The Central Hospital of Jinzhou, Jinzhou, Liaoning 121000; ${ }^{3}$ Department of Ophthalmology, \\ The First Affiliated Hospital of China Medical University, Shenyang, Liaoning 110000, P.R. China
}

Received April 17, 2016; Accepted March 24, 2017

DOI: $10.3892 / \mathrm{etm} .2017 .5565$

\begin{abstract}
The aim of the present study was to evaluate the efficacy of combination treatment with intravitreal ranibizumab (IVR) injection and photodynamic therapy (PDT) for polypoidal choroidal vasculopathy (PCV). A total of 64 patients with PCV were included in the present study, which were divided into the IVR monotherapy group (Group A) and combination treatment groups (Groups B-D) with different treatment intervals. All subjects were followed-up at 1, 3, 6 and 12 months following treatment, and subjected to the detection of best-corrected visual acuity (BCVA) and central foveal thickness (CFT). Compared with the monotherapy group, more significant BCVA improvement was observed for the combination treatment groups, with the most evident effect exhibited in Group C. At the end of the follow-up period, visual acuity improvement rates were markedly elevated in the combination treatment groups, as compared with the monotherapy group. According to optical coherence tomography, the CFT for the combination treatment groups was thinner than the monotherapy group. Among the combination groups, CFT improvement for Group C was superior to other groups. Fundus angiography demonstrated that, compared with monotherapy, combination treatment may significantly promote the regression and prevent the recurrence of polyps and BVN. The most efficient effectiveness was observed for Group C. In addition, combination treatment may significantly reduce the IVR injection numbers required to treat PCV. Patients receiving combination treatment with IVR injection and PDT have greater vision improvements, reduced macular degeneration and decreased injection numbers. Combination therapy may
\end{abstract}

Correspondence to: Professor Zheli Liu, Department of Ophthalmology, The First Affiliated Hospital of China Medical University, 155 Nanjing North Road, Shenyang, Liaoning 110000, P.R. China

E-mail: 1ijia820323@163.com

Key words: polypoidal choroidal vasculopathy, intravitreal ranibizumab injection, photodynamic therapy, combination therapy therefore, represent an effective and safe therapeutic strategy for PCV clinical treatment.

\section{Introduction}

Polypoidal choroidal vasculopathy (PCV) is characterized by branching choroidal networks with polyp-like aneurysmal dilation, which are clearly detected by indocyaninegreen angiography (ICGA) $(1,2)$. Clinically, these polyps are typically an orange or red color and either isolated or connected with a branching vascular network (BVN) (3). In PCV pathogenesis, the polyps are associated with serous exudation and/or hemorrhage, which may lead to retinal pigment epithelium (RPE) detachment. In certain cases, it may also result in subretinal fluid (SRF), accompanied by a detached neurosensory retina (4). Without proper and timely treatment, patients with PCV would suffer from recurrent episodes of hemorrhage and exudative maculopathy in the eye, potentially resulting in irreversible visual loss. Current treatments primarily include pharmaceutical therapy, laser treatment, and surgery (5).

A number of therapeutic strategies have been reported for PCV clinically $(6,7)$. For example, anti-vascular endothelial growth factor (VEGF) injection may reduce lesion leakage and decrease high perfusion in patients with PCV (3). Furthermore, photodynamic therapy (PDT) has also been demonstrated to effectively seal the blood vessels in PCV, and subsequently limit lesion development $(6,7)$. However, long-term follow-up observations have indicated a higher recurrence rate and retinal hemorrhage incidence following PDT, and therefore repeated treatments are required $(8,9)$. By contrast, combination therapy with intravitreal ranibizumab (IVR) injection and PDT has been demonstrated to have more satisfactory outcomes, which may significantly reduce the incidence of complications $(10,11)$. The Everest study was a prospective, randomized study comparing the treatment outcomes between IVR monotherapy and combination therapy with IVR and PDT for PCV. The study inferred that verteporfin PDT combined with ranibizumab $(0.5 \mathrm{mg})$ or alone is superior to the ranibizumab monotherapy in achieving complete regression of polyps in patients with symptomatic macular PCV, over a 6-month period (12).

In the present study, the efficacy and safety of combination therapy with IVR injection and PDT for the treatment of PCV 
were evaluated. A total of $64 \mathrm{PCV}$ cases were included and divided into IVR monotherapy group and three combination treatment (IVR + PDT) groups with different treatment intervals. All subjects were followed-up at 1, 3,6 and 12 months. The results from the best-corrected visual acuity (BCVA) and central foveal thickness (CFT) evaluation were analyzed and compared.

\section{Materials and methods}

Study subjects and grouping. A total of 64 patients (64 eyes; 37 male and 27 female subjects, with a mean age of $67.87 \pm 5.49$ years) with PCV, who were admitted to the First Affiliated Hospital of China Medical University (Liaoning, China) between March 2013 and August 2015, were included in the current study. All patients had been subjected to comprehensive baseline ophthalmic examination every month for 1 year previous to the study. Inclusion criteria were as follows: i) Fundus examination indicating swelling and/or red lesions; ii) treatment-naïve PCV, as characterized by the presence of polyps or BVN; iii) >50 years of age; and iv) greatest linear dimension of $\leq 5,400 \mathrm{~mm}$ (13). Exclusion criteria included: i) Prior treatment with intravitreal injection of either anti-VEGF or verteporfin; ii) vitreous hemorrhage; iii) extensive subretinal hemorrhage preventing proper imaging or PDT from being performed; iv) scar tissue area accounting for $\geq 50 \%$ of the lesion; and v) accompanied with other retinal diseases including diabetic retinopathy (14). Prior written and informed consent were obtained from each patient and the study was approved by the Ethics Review Board of the First Affiliated Hospital of China Medical University.

Although the same inclusion and exclusion criteria for PCV were applied, the patients' conditions differed from each other. Due to a lack of uniform treatment protocol for PCV, different therapeutic treatments would be performed for different patients, including the treatment intervals for the combination therapy. In the Department of Ophthalmology, the First Affiliated Hospital of China Medical University (Shenyang, China), the usual treatment intervals were set as 3, 7 and 10 days. In the present study, thorough communication was conducted with each patient with PCV in advance, and the therapeutic regimens were determined according to the patients' conditions and experts' experience. Accordingly, these patients were randomly divided into four groups: IVR monotherapy group (Group A) and three combination treatment (IVR + PDT) groups (Groups B-D) with different treatment intervals of 3, 7 and 10 days, respectively.

In the monotherapy group $(n=16)$, there were 9 male and 7 female subjects, with a mean age of $66.12 \pm 5.14$ years and a mean $\log$ minimum resolution angular (MAR) BCVA of $0.67 \pm 0.22$ In Group B $(n=16)$, there were 10 male and 6 female subjects, with a mean age of $65.78 \pm 6.32$ years and a mean $\log$ MAR BCVA of $0.69 \pm 0.15$. In Group C $(n=17)$, there were 9 male and 8 female subjects, with a mean age of $69.26 \pm 4.35$ years and a mean $\log$ MAR BCVA of $0.71 \pm 0.31$. In Group D $(n=15)$, there were 9 male and 6 female subjects, with a mean age of $70.35 \pm 6.14$ years and a mean $\log$ MAR BCVA of $0.62 \pm 0.28$.

Ophthalmic treatment and examination. For the IVR monotherapy group (Group A), patients were subjected to vitreous cavity injection of $0.5 \mathrm{mg}$ ranibizumab. On the next day, visual acuity and intraocular pressure were assessed by routine exanimations and the slit-lamp examination was performed (15). Levofloxacin eye drops were administered to these patients four times per day, for three consecutive days. For the IVR monotherapy group (Group A), the patients were subjected to monthly injections, for three consecutive months. Retreatment was considered according to the protocol in the PrONTO study (14). An additional intravitreal injection was administered if any of the following changes were observed: i) Visual acuity loss of at least five letters, with optical coherence tomography (OCT) evidence of fluid in macula; ii) an increase in OCT central retinal thickness of $\geq 100 \mu \mathrm{m}$; iii) enlarged pigment epithelial detachment; iv) new macular hemorrhage; or v) persistent fluid on OCT 1 month following previous treatment. A total of 15, 7, 3 and 4 patients required addition intravitreal injection in groups A, B, C and D, respectively.

For the combination treatment groups (Groups B-D), the patients were subjected to an intravitreal injection of $0.5 \mathrm{mg}$ ranibizumab, followed by a session of PDT. For Group B $(n=16)$, the treatment interval between IVR injection and PDT was set as 3 days. For Groups $C(n=17)$ and $D(n=15)$, the treatment intervals were set to be 7 and 10 days, respectively. PDT was performed using a 689-nm diode laser unit (Visulas PDT system 690S; Carl Zeiss, Dublin, CA, USA) following an injection of verteporfin $(10.5 \mathrm{mg})$, according to the guidelines for age-related macular degeneration (16). Following the initial treatment regimen, follow-up evaluations were scheduled to be at 1-month intervals. Fundus angiography (FFA/ICGA) was performed on each patient prior to and 3 months after treatment. Additional angiography was performed if necessary. Additional PDT was considered when the intravitreal injection of ranibizumab exhibited minimal effectiveness and polypoidal lesions with exudative changes were detected in ICGA $(17,18)$.

Statistical analysis. Data were expressed as mean \pm standard deviation. SPSS version 16.0 software (SPSS, Inc., Chicago, IL, USA) was used for statistical analysis. Following a variance homogeneity assessment, two-way independent-sample t-test was used for group comparison, and single-factor analysis of variance was performed for comparisons of prior to and following treatment. $\mathrm{P}<0.05$ was considered to indicate a statistically significant difference.

\section{Results}

$B C V A$. Analysis of $\log$ MAR BCVA indicates that, compared with the $\log$ MAR BCVA prior to treatment, all patients in the monotherapy and combination treatment groups improved following treatment. As presented in Fig. 1, more significant BCVA improvement was observed for the combination treatment groups, compared with the monotherapy group $(\mathrm{P}<0.05)$. Among the combination therapy groups, compared with Groups B and D, Group C was associated with the most efficient therapeutic effect regarding the BCVA improvement, specifically 3, 6 and 12 months following treatment.

At the end of the follow-up period, the logMAR BCVA was significantly improved compared with logMAR BCVA prior to treatment, in all the monotherapy and combination treatment 


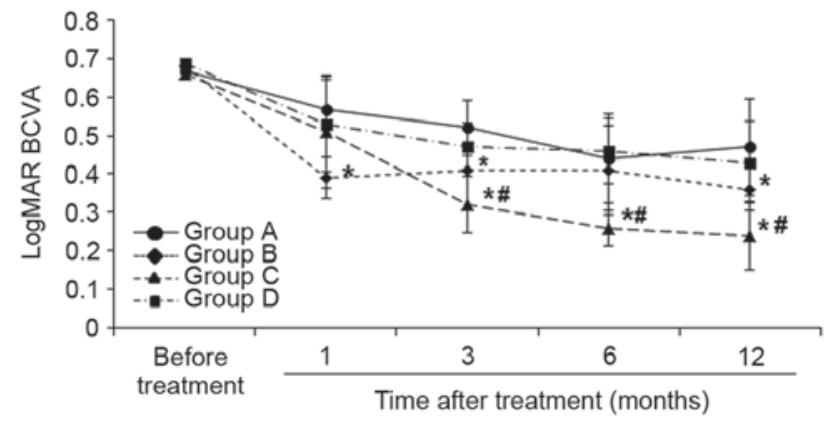

Figure 1. BCVA of patients with PCV subjected to monotherapy and combination treatments. The eyes of patients with PCV were subjected to monotherapy (Group A) or combination treatment with the treatment intervals of 3 days (Group B), 7 days (Group C) or 10 days (Group D), respectively. Mean $\log$ MAR BCVA values for the monotherapy and combination treatment groups were obtained and compared at 1,3,6 and 12 months following treatment. ${ }^{*} \mathrm{P}<0.05$ vs. Group A; ${ }^{\#} \mathrm{P}<0.05$ vs. Groups B and D. Data are presented as the mean \pm standard deviation. BCVA, best-corrected visual acuity; PCV, polypoidal choroidal vasculopathy; logMAR, log minimum resolution angular.

groups ( $\mathrm{P}<0.05 ;$ Fig. 1$)$. The results of the present study demonstrated that, in the monotherapy group (Group A), the visual acuity improvement rate was $18.8 \%$ (3/16). Compared with the monotherapy group, the visual acuity improvement rates were markedly elevated in the combination treatment groups. Visual acuity improvement rates for Groups B-D were 25\% (4/16), 29.4\% (5/17), and 26.7\% (4/15), respectively (Fig. 2). These results suggest that, compared with the monotherapy group, a more significant improvement in visual acuities was observed for the combination treatment groups, with Group C exhibiting the best efficiency.

OCT. According to OCT, prior to treatment, the mean CFT values for Groups A-D were 456.58 $\pm 46.33,471.49 \pm 38.52$, $468.71 \pm 29.16$, and $462.54 \pm 2668 \mu \mathrm{m}$, respectively, with no statistically significant differences between groups $(\mathrm{P}>0.05)$. As presented in Fig. 3, the CTF improvements for the combination treatment groups (Groups B-D) were typically superior to the monotherapy group (Group A) following treatment. Among the combination groups, CFT improvement for Group $C$ was significantly superior to Groups $B$ and $D$, at 1,3 , 6 and 12 months following treatment $(\mathrm{P}<0.05)$. These results suggest that, combination treatments are more effective in reducing CTF than monotherapy, with the most efficient effect observed for Group C.

FFA/ICGA. According to the FFA/ICGA, the results of the present study indicate that, in Group A, polyps were reduced in 7 eyes (43.8\%) and branching vascular networks (BVN) were decreased in 5 eyes (31.3\%), while the recurrence of polyps/BVN was confirmed in 4 eyes $(25.0 \%)$. Therapeutic efficiency was markedly promoted for the combination treatments. In Groups B-D, polyps were reduced in 8 (50\%), $9(52.9 \%)$ and $6(40.0 \%)$ eyes, respectively. Furthermore, BVN was decreased in 7 (43.8\%), 8 (47.1\%) and 7 (46.7\%) eyes in Groups B-D, respectively. By contrast, recurrence of polyps/BVN was observed in $1(6.3 \%), 0(0.0 \%)$ and $2(13.3 \%)$ eyes, for Groups B-D, respectively. As presented in Figs. 4 and 5, FFA/ICGA indicated decreased lesion areas,

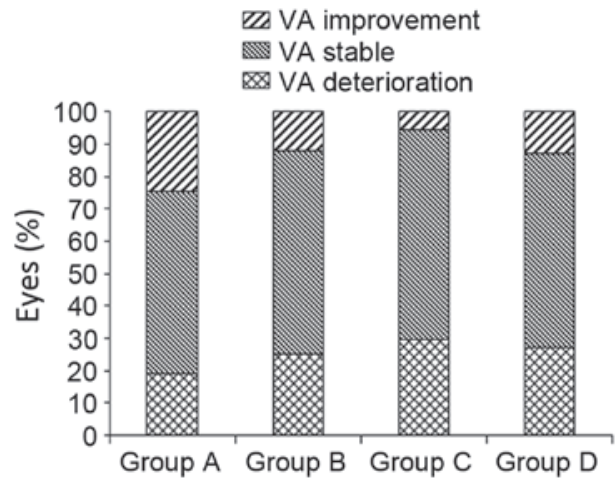

Figure 2. VA analysis for the monotherapy and combination groups. At the end of the follow-up period, the visual acuities were analyzed and compared for the monotherapy and combination treatment groups. VA, visual acuity; Group A, monotherapy group; Group B, combination treatment at 3 days; Group C, combination treatment at 7 days; Group D, combination treatment at 10 days.

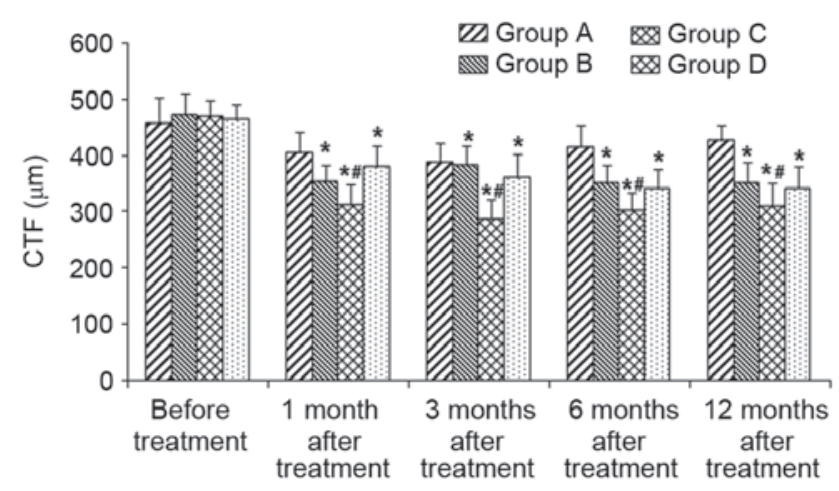

Figure 3. CFT changes for patients with PCV subjected to monotherapy and combination treatments. The eye of patients with PCV were subjected to monotherapy (Group A) and combination treatments with treatment intervals of 3 days (Group B), 7 days (Group C) and 10 days (Group D), respectively. The CFT for the monotherapy and combination treatment groups was detected with OCT 1, 3, 6 and 12 months following treatment. ${ }^{*} \mathrm{P}<0.05$ vs. Group A; ${ }^{\text {P }}<0.05$ vs. Group B and D. Data are presented as the mean \pm standard deviation. CFT, central foveal thickness; PCV, polypoidal choroidal vasculopathy; OCT, optical coherence tomography.

reduced bleeding and exudation, and alleviated pigment epithelium detachment following the combination treatment. Compared with monotherapy, combination treatment may significantly promote the regression and prevent recurrence of polyps/BVN in patients with PCV. Superior results were, again, observed for Group C.

$I V R$ injection numbers and adverse reactions. Influence of IVR injection numbers and the adverse reactions were also analyzed. The IVR injection number for Group A was $3.79 \pm 0.81$, which was significantly higher than the combination groups $(1.81 \pm 0.56,1.72 \pm 0.68$ and $1.79 \pm 0.74$ for Groups B-D, respectively; all $\mathrm{P}<0.05)$. By contrast, no significant adverse reactions (such as retinal detachment, sustained high intraocular pressure, retinal hole and endophthalmitis) were observed for all groups, during the treatment course and in the follow-up period. These results suggest that, compared with monotherapy, combination treatment may significantly reduce the number of required IVR injections in the treatment of PCV. 
A

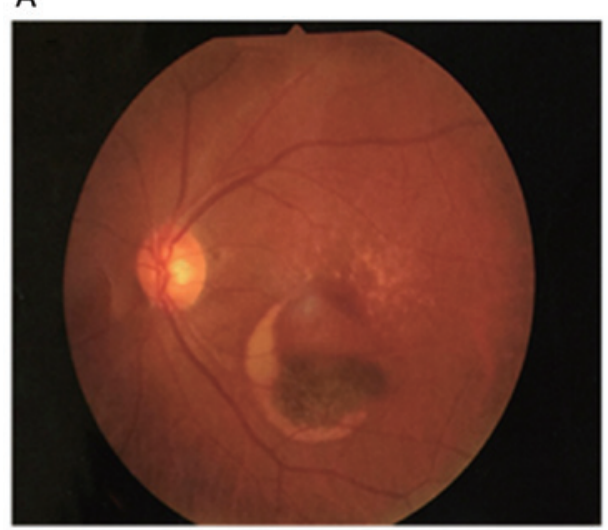

B

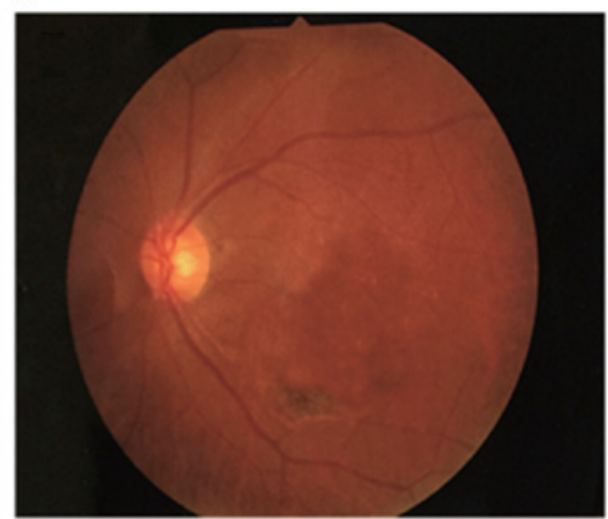

Figure 4. Fundus photography images of the left eye of a 74-year-old male patient subjected to combination treatment. (A) Prior to treatment, hemorrhage and white exudation were observed, with a visual acuity of 0.1. (B) At 12 months following treatment, the bleeding and exudation were markedly reduced, with a visual acuity of 0.7 .
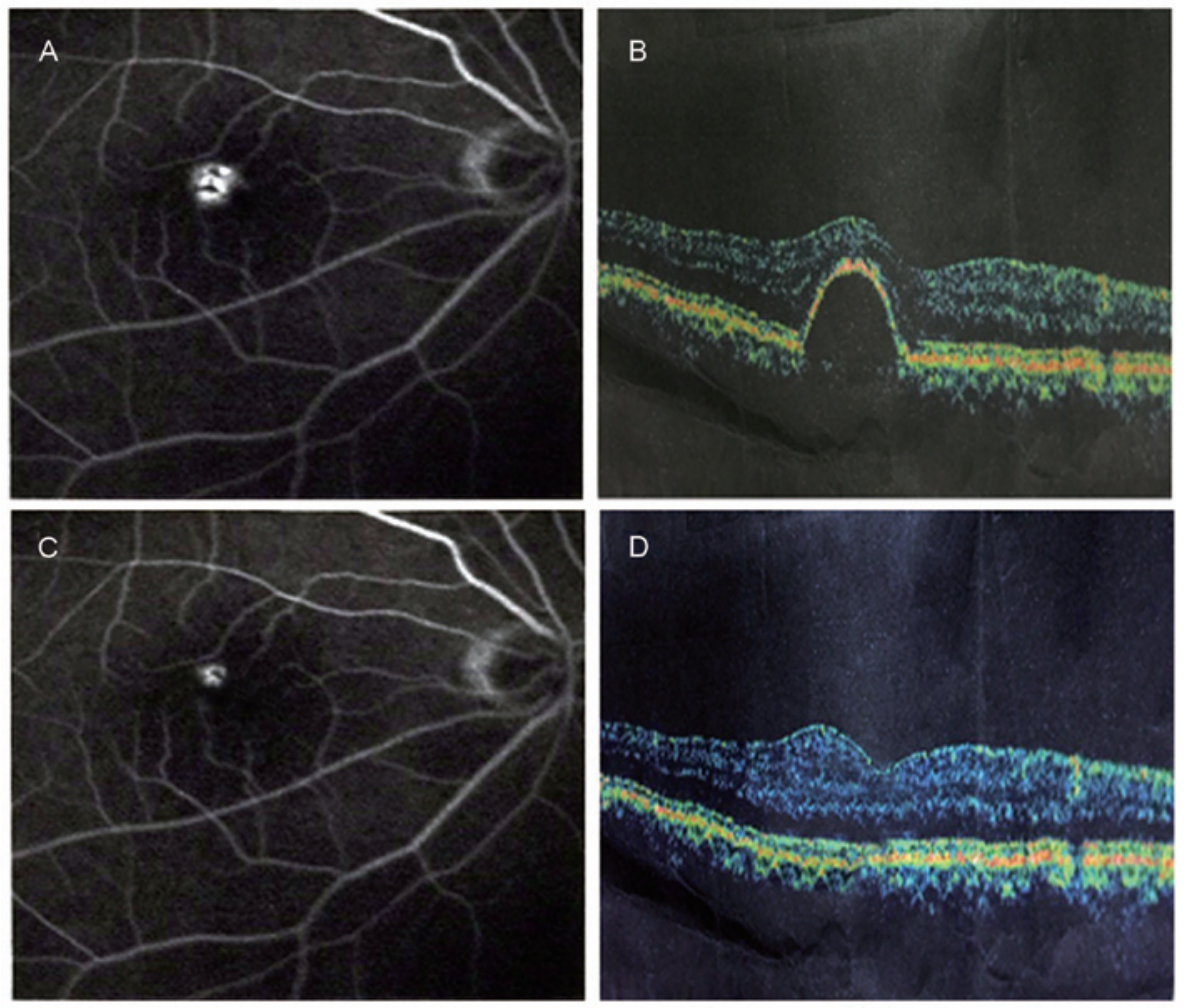

Figure 5. ICGA images of the right eye of a 76-year-old male subjected to combination treatment. Prior to treatment (A) ICGA indicated fluorescent leakage and (B) OCT indicated retinal pigment epithelial detachment on the macula, with a visual acuity of 0.2. At 6 months post-treatment (C) the fluorescent leakage was significantly decreased and (D) the retinal pigment epithelial detachment was reduced, with a visual acuity of 0.6. ICGA, indocyaninegreen angiography; OCT, optical coherence tomography.

\section{Discussion}

At present, PDT is one of the major therapeutic treatments for PCV clinically. However, retinal hemorrhage, RPE tear and other complications have been reported to be associated with PDT in the treatment of PCV (9). Ranibizumab may reduce lesion leakage and improve or stabilize visual acuity in patients with PCV (19). Therefore, the present study hypothesized that PDT combined with IVR injection may improve visual acuity, reduce polyps, decrease the incidence of complications and achieve a more satisfactory therapeutic effect in the treatment of PCV.
The results of the current study indicate that the $\log$ MAR BCVA following treatment was improved for all monotherapy and combination groups compared with the logMAR BCVA prior to treatment, which were in line with previous findings published by Lai et al (20). Furthermore, the results of the current study indicated that, compared with monotherapy, combination treatments may more effectively improve the visual acuities of patients with PCV, which were consistent with the results of Sato et al (21). Among the combination groups, the greatest therapeutic effect was observed for Group C. At the end of the follow-up period, combination treatments 
significantly improved and stabilized visual acuity in patients with PCV, more efficiently than monotherapy. In line with previous findings $(22,23)$, Group $C$ was associated with the most efficient therapeutic effects in the treatment of PCV. However, a previous study has indicated that no clear difference was observed in the visual acuity 6 months after combination treatment in patients with PCV (24). Rouvas et al (25) reported that there were no significant differences in BCVA between the control and treatment groups 12 months after treatment. This contradiction may be due to the differential ethnic backgrounds of PCV cases and differences in drug sensitivity. The results of the present study indicate that, at the end of therapy, the combination treatments were more effective in improving the patients' conditions. Notably, the CFT was significantly reduced compared with the monotherapy group. Furthermore, FFA/ICGA indicated a decreased lesion area, reduced polyps/BVN and improved pigment epithelium detachment. In line with a previous result, Group C exhibited superior results.

In previous studies, there have been a number of variations in the treatment regimens for combination therapy with IVR injection and PDT for PCV $(25,26)$. In the Everest study, IVR injection and PDT in the combination treatment were performed on the same day (12). By contrast, PDT was performed 4-7 days after the initial IVR injection in another study (26). In the present study, the combination treatment groups were divided into three groups (Groups B-D), with different treatment intervals (PDT was performed at 3,7 and 10 days, respectively, following the initial IVR injection). According to the results of the current study, the treatment interval of 7 days (Group C) was most efficient and suitable for the combination treatment of PCV. With regard to the IVR injection numbers, three consecutive IVR injections were administered at 4-week intervals in the present study and in the Everest study (25). The results of the current study demonstrated that combination treatments significantly reduced the injection numbers compared with monotherapy. Furthermore, the peak therapeutic effects of IVR injection were achieved, which was also the most appropriate timing for PDT, with the smallest irradiation spot and energy, minimizing the side effects and damage to the choroid and retina. Therefore, the combination of IVR injection and PDT with the time interval of 7 days demonstrated the best treatment efficacy and stability. However, the treatment efficiency may be compromised when the time intervals were too short or too long, while retinal damages induced by PDT would also be enhanced, reducing the therapeutic effectiveness of the combination treatment. This may be explained by the cumulative effect of IVR injection and PDT. Further in-depth studies are required to investigate the optimal combination regime for the treatment of PCV.

In conclusion, the results of the present study demonstrated that combination treatment with IVR injection and PDT may improve the therapeutic effects for PCV. Compared with the monotherapy group, the combination treatments may significantly improve and stabilize visual acuity, reduce $\mathrm{CTF}$ and promote the regression or prevent the recurrence of polyps/BVN, in patients with PCV. Furthermore, the most effective protocol was Group C, when the treatment interval between IVR injection and PDT was set as 7 days. In addition, combination treatments may significantly reduce the IVR injection numbers in the treatment of PCV. The results of the current study suggest that the combination treatment of IVR injection and PDT represents an effective and safe therapeutic strategy for PCV, which may contribute to the disease treatment clinically. Further prospective, randomized controlled studies, with enlarged samples and multiple centers, are required to investigate the optimal treatment option for patients with PCV.

\section{Acknowledgements}

The present study was supported by the Joint Grant of Liaoning Province Science and Technology Department (grant no. 2015020351).

\section{References}

1. Cheng CK, Peng CH, Chang CK, Hu CC and Chen LJ: One-year outcomes of intravitreal bevacizumab (avastin) therapy for polypoidal choroidal vasculopathy. Retina 31: 846-856, 2011.

2. Cho HJ, Kim JW, Lee DW, Cho SW and Kim CG: Intravitreal bevacizumab and ranibizumab injections for patients with polypoidal choroidal vasculopathy. Eye (Lond) 26: 426-433, 2012.

3. Koh AH; Expert PCV Panel, Chen LJ, Chen SJ, Chen Y, Giridhar A, Iida T, Kim H, Yuk Yau Lai T, Lee WK, et al: Polypoidal choroidal vasculopathy: Evidence-based guidelines for clinical diagnosis and treatment. Retina 33: 686-716, 2013.

4. Sho K, Takahashi K, Yamada H, Wada M, Nagai Y, Otsuji T, Nishikawa M, Mitsuma Y, Yamazaki Y, Matsumura M and Uyama M: Polypoidal choroidal vasculopathy: Incidence, demographic features and clinical characteristics. Arch Ophthalmol 121: 1392-1396, 2003.

5. Akaza E, Yuzawa M and Mori R: Three-year follow-up results of photodynamic therapy for polypoidal choroidal vasculopathy. Jpn J Ophthalmol 55: 39-44, 2011.

6. Miura M, Makita S, Iwasaki T and Yasuno Y: Three-dimensional visualization of ocular vascular pathology by optical coherence angiography in vivo. Invest Ophthalmol Vis Sci 52: 2689-2695, 2011.

7. Hata M, Tsujikawa A, Miyake M, Yamashiro K, Ooto S, Oishi A, Nakata I, Takahashi A and Yoshimura N: Two-year visual outcome of polypoidal choroidal vasculopathy treated with photodynamic therapy combined with intravitreal injections of ranibizumab. Graefes Arch Clin Exp Ophthalmol 253: 189-197, 2015.

8. Yoshida Y, Kohno T, Yamamoto M, Yoneda T, Iwami H and Shiraki K: Two-year results of reduced-fluence photodynamic therapy combined with intravitreal ranibizumab for typical age-related macular degeneration and polypoidal choroidal vasculopathy. Jpn J Ophthalmol 57: 283-293, 2013.

9. Sakurai M, Baba T, Kitahashi M, Yokouchi H, Kubota-Taniai M, Bikbova G, Oshitari T and Yamamoto S: One-year results of intravitreal ranibizumab combined with reduced-fluence photodynamic therapy for polypoidal choroidal vasculopathy. Clin Ophthalmol 8: 235-241, 2014.

10. Nicolo M, Eandi CM, Alovisi C, Grignolo FM, Traverso CE, Musetti D and Cardillo Piccolino F: Half-fluence versus half-dose photodynamic therapy in chronic central serous chorioretinopathy. Am J Ophthalmol 157: 1033-1037, 2014.

11. Sagong M, Lim S and Chang W: Reduced-fluence photodynamic therapy combined with intravitreal bevacizumab for polypoidal choroidal vasculopathy. Am J Ophthalmol 153: 873-882.e2, 2012.

12. Koh A, Lee WK, Chen LJ, Chen SJ, Hashad Y, Kim H, Lai TY, Pilz S, Ruamviboonsuk P, Tokaji E, et al: EVEREST study: Efficacy and safety of verteporfin photodynamic therapy in combination with ranibizumab or alone versus ranibizumab monotherapy in patients with symptomatic macular polypoidal choroidal vasculopathy. Retina 32: 1453-1464, 2012.

13. Miki A, Honda S, Kojima H, Nishizaki M, Nagai T, Fujihara M, Uenishi M, Kita M, Kurimoto Y and Negi A; Hyogo Macular Disease Study Group: Visual outcome of photodynamic therapy for typical neovascular age-related macular degeneration and polypoidal choroidal vasculopathy over 5 years of follow-up. Jpn J Ophthalmol 57: 301-307, 2013.

14. Yamashita A, Shiraga F, Shiragami C, Shirakata Y and Fujiwara A: Two-year results of reduced-fluence photodynamic therapy for polypoidal choroidal vasculopathy. Am J Ophthalmol 155: 96-102.e2, 2013. 
15. Watanabe R, Nakazawa $\mathrm{T}$ and Fuse N: Observation of posterior corneal vesicles with in vivo confocal microscopy and anterior segment OCT. Clin Ophthalmol 4: 1243-1247, 2010

16. Ricci F, Calabrese A, Regine F, Missiroli F and Ciardella AP: Combined reduced fluence photodynamic therapy and intravitreal ranibizumab for polypoidal choroidal vasculopathy. Retina 32: 1280-1288, 2012.

17. Sato T, Kishi S, Matsumoto $\mathrm{H}$ and Mukai R: Comparisons of outcomes with different intervals between adjunctive ranibizumab and photodynamic therapy for polypoidal choroidal vasculopathy. Am J Ophthalmol 156: 95-105.e1, 2013.

18. Wong IY, Shi X, Gangwani R, Zhao P, Iu LP, Li Q, Ng A and Li X: 1-year results of combined half-dose photodynamic therapy and ranibizumab for polypoidal choroidal vasculopathy. BMC Ophthalmol 15: 66, 2015.

19. Hikichi T, Higuchi M, Matsushita T, Kosaka S, Matsushita R, Takami K, Ohtsuka H and Ariga H: One-year results of three monthly ranibizumab injections and as-needed reinjections for polypoidal choroidal vasculopathy in Japanese patients. Am J Ophthalmol 154: 117-124.e1, 2012.

20. Lai TY, Lee GK, Luk FO and Lam DS: Intravitreal ranibizumab with or without photodynamic therapy for the treatment of symptomatic polypoidal choroidal vasculopathy. Retina 31 : 1581-1588, 2011.

21. Sato T, Kishi S, Matsumoto H and Mukai R: Combined photodynamic therapy with verteporfin and intravitreal bevacizumab for polypoidal choroidal vasculopathy. Am J Ophthalmol 149: 947-954.e1, 2010.
22. Nowak-Sliwinska $P$, van den Bergh H, Sickenberg $M$ and Koh AH: Photodynamic therapy for polypoidal choroidal vasculopathy. Prog Retin Eye Res 37: 182-199, 2013.

23. Chung WH, van Dijk EH, Mohabati D, Dijkman G, Yzer S, de Jong EK, Fauser S, Schlingemann RO, Hoyng CB, Boon CJ: Neovascular age-related macular degeneration without drusen in the fellow eye: Clinical spectrum and therapeutic outcome. Clin Ophthalmol 11: 63-70, 2016.

24. Kang HM and Koh HJ: Two-year outcome after combination therapy for polypoidal choroidal vasculopathy: comparison with photodynamic monotherapy and anti-vascular endothelial growth factor monotherapy. Ophthalmologica 231: 86-93, 2014.

25. Rouvas AA, Papakostas TD, Ntouraki A, Douvali M, Vergados I and Ladas ID: Photodynamic therapy, ranibizumab and ranibizumab with photodynamic therapy for the treatment of polypoidal choroidal vasculopathy. Retina 31: 464-474, 2011

26. Byon IS, Kwon HJ, Kim SI, Shin MK, Park SW and Lee JE: Reduced-fluence photodynamic therapy in polypoidal choroidal vasculopathy nonresponsive to ranibizumab. Ophthalmic Surg Lasers Imaging Retina 45: 534-541, 2014. 